PROCEEDINGS OF THE

AMERICAN MATHEMATICAL SOCIETY

Volume 127, Number 6, Pages 1745-1751

S 0002-9939(99)04911-4

Article electronically published on February 11, 1999

\title{
AN EXTENSION OF THE INTEGRAL TEST
}

\author{
ANA LÍA DURÁN AND RICARDO ESTRADA \\ (Communicated by Frederick W. Gehring)
}

\begin{abstract}
We identify a class of functions for which an extension of the well known integral test holds, namely, the series $\sum_{k=1}^{\infty} \phi(k)$ is Cesàro summable if and only if the integral $\int_{1}^{\infty} \phi(x) d x$ is. We also give the corresponding multidimensional results.
\end{abstract}

\section{INTRODUCTION}

The integral test for the convergence of series is a standard topic in any introductory calculus sequence. In its basic form it says that if $\phi$ is a continuous positive decreasing function defined in the interval $[1, \infty)$, then the series

$$
\sum_{k=1}^{\infty} \phi(k)
$$

and the integral

$$
\int_{1}^{\infty} \phi(x) d x
$$

are both convergent or both divergent. That the test fails if $\phi$ is not decreasing is easy to see by simple examples, such as $\phi(x)=\sin \pi x$ for which (1.1) converges but (1.2) does not, or $\phi(x)=\sum_{n=1}^{\infty}\left(1+n^{4}(x-n)^{2}\right)^{-1}$ for which (1.2) converges but (1.1) does not.

The purpose of this note is to show that under mild conditions on $\phi$, that assure that it does not oscillate too fast, the integral test remains valid if (1.1) and (1.2) are understood in the sense of Cesàro summability.

Our results are another instance of the important intuitive idea that sums $\sum \phi(k)$ can be approximated by integrals $\int \phi(x) d x$ and conversely. Other instances include the well-known Euler-Maclaurin formula [6], [8] or the recent result [2] according to which under mild assumptions on $\phi$, the zeta-function $\sum_{k=1}^{\infty} \phi(k)^{s}$ admits an analytic continuation to the same region in the $s$-plane as the integral $\int_{1}^{\infty} \phi(x)^{s} d x$.

Our analysis is based on a study of the distribution

$$
\sigma(x)=\sum_{k=1}^{\infty} \delta(x-k)-H(x-1)
$$

Received by the editors September 12, 1997.

1991 Mathematics Subject Classification. Primary 26A06, 40A10, 46F10.

Key words and phrases. Integral, series, summability.

(C)1999 American Mathematical Society 
where $\delta(x-\xi)$ is the Dirac delta function concentrated at the point $\xi$ while $H(x)$ is the Heaviside function: $H(x)=0, x<0 ; H(x)=1, x>0$, so that $H(x-1)$ is the characteristic function of the interval $[1, \infty)$. Indeed,

$$
\langle\sigma(x), \phi(x)\rangle=\sum_{k=1}^{\infty} \phi(k)-\int_{1}^{\infty} \phi(x) d x
$$

and thus the question of the equivalence of (1.1) and (1.2) becomes the question of finding the spaces of test functions $\mathcal{A}$ for which the distribution $\sigma$ belongs to the dual space $\mathcal{A}^{\prime}$.

We also consider multidimensional versions of (1.3), such as

$$
\sigma_{d}(\mathbf{x})=\sum_{\mathbf{k} \in \mathbb{Z}^{d}} \delta(\mathbf{x}-\mathbf{k})-1
$$

and, in this way, we obtain versions of the integral test in $\mathbb{R}^{d}$.

\section{Preliminaries}

In this section we discuss some results from the theory of distributions that will be needed in our analysis. For the basic facts on the theory of distributions, in particular, on the standard spaces $\mathcal{D}^{\prime}$ and $\mathcal{S}^{\prime}$, we refer to the textbooks [9], [10].

The spaces needed in this article are the spaces $\mathcal{G}_{\beta}\left(\mathbb{R}^{d}\right)$ that we now explain. Let $\beta \in \mathbb{R}$ and $q \in \mathbb{R}$. A function $\phi$ defined in $\mathbb{R}^{d}$ belongs to $\mathcal{G}_{\beta, q}\left(\mathbb{R}^{d}\right)$ if $\phi$ is smooth and if for each multi-index $\mathbf{k} \in \mathbb{N}^{d}$ it satisfies $\mathbf{D}^{\mathbf{k}} \phi(|\mathbf{x}|)=O\left(|\mathbf{x}|^{q-\beta|\mathbf{k}|}\right)$ as $|\mathbf{x}| \rightarrow \infty$. Here we use the standard notation: if $\mathbf{k}=\left(k_{1}, \ldots, k_{d}\right)$, then $\mathbf{D}^{\mathbf{k}}=\frac{\partial^{|\mathbf{k}|}}{\partial x_{1}^{k_{1}} \ldots \partial x_{d}^{k_{d}}}$, $|\mathbf{k}|=k_{1}+\cdots+k_{d}$. A topology is obtained by considering the family of seminorms

$$
\|\phi\|_{q, \mathbf{k}}=\sup \left\{\rho_{q-\beta|\mathbf{k}|}(|\mathbf{x}|)\left|\mathbf{D}^{\mathbf{k}} \phi(\mathbf{x})\right|: \mathbf{x} \in \mathbb{R}^{d}\right\},
$$

where $\rho_{q}(r)=1$ for $0 \leq r \leq 1$ and $\rho_{q}(r)=r^{-q}$ for $r \geq 1$. The space $\mathcal{G}_{\beta}\left(\mathbb{R}^{d}\right)$ is the inductive limit of the spaces $\mathcal{G}_{\beta, q}\left(\mathbb{R}^{d}\right)$ as $q \rightarrow \infty$.

If $\beta=1$, we obtain the space $\mathcal{K}=\mathcal{G}_{1}$ of so-called GLS symbols [7]. The dual space $\mathcal{K}^{\prime}$ is fundamental in the theory of distributional asymptotic expansions [6] and in the theory of summability of distributional evaluations [4], [5]. Indeed, as we explain below, if $f \in \mathcal{K}^{\prime}\left(\mathbb{R}^{d}\right)$ and $\phi \in \mathcal{K}\left(\mathbb{R}^{d}\right)$, then the evaluation $\langle f(\mathbf{x}), \phi(\mathbf{x})\rangle$ can be computed as a limit in the Cesàro sense.

The idea of interpreting distributional evaluations as Cesàro limits is best introduced by using the order symbols $O\left(x^{\alpha}\right)$ and $o\left(x^{\alpha}\right)$ in the Cesàro sense. If $f \in \mathcal{D}^{\prime}(\mathbb{R})$ and $\alpha \in \mathbb{R} \backslash\{-1,-2,-3, \ldots\}$, we say that $f(x)=O\left(x^{\alpha}\right)$ as $x \rightarrow \infty$ in the Cesàro sense, and write

$$
f(x)=O\left(x^{\alpha}\right)(C) \quad \text { as } x \rightarrow \infty,
$$

if there exists $N \in \mathbb{N}$ such that each primitive $F$ of order $N$ of $f$, i.e., $F^{(N)}=f$, is an ordinary function for large arguments and satisfies the ordinary order relation

$$
F(x)=p(x)+O\left(x^{\alpha+N}\right) \quad \text { as } x \rightarrow \infty,
$$

for a suitable polynomial $p$ of degree $N-1$ at most. A similar definition applies to the little $o$ symbol.

We say that a distribution $f \in \mathcal{D}^{\prime}(\mathbb{R})$ has the limit $L$ in the Cesàro sense as $x \rightarrow \infty$, and write $\lim _{x \rightarrow \infty} f(x)=L(C)$, if $f(x)=L+o(1)(C)$ as $x \rightarrow \infty$. 
Distributional evaluations are treated as follows. Suppose first that $\operatorname{supp} f$ is bounded on the left, and consider the evaluation $\langle f(x), \phi(x)\rangle$. Let $g(x)$ be the first order primitive of $\phi(x) f(x)$ with support bounded on the left. We say that the evaluation $\langle f(x), \phi(x)\rangle$ exists in the Cesàro sense and equals $L$, and write

$$
\langle f(x), \phi(x)\rangle=L(C)
$$

if $\lim _{x \rightarrow \infty} g(x)=L(C)$. The case when the support of $f$ is bounded on the right is similar and the general case is handled by writing $f=f_{1}+f_{2}$ with supp $f_{1}$ bounded on the left and $\operatorname{supp} f_{2}$ bounded on the right. It is easy to see that the Cesàro limit of the evaluation is independent of the decomposition.

Theorem 2.1. Let $f \in \mathcal{K}^{\prime}(\mathbb{R})$ and $\phi \in \mathcal{K}(\mathbb{R})$. Then the evaluation $\langle f(x), \phi(x)\rangle$ exists in the Cesàro sense.

Proof. See [4].

In the multidimensional case we consider Cesàro summability by spherical means. The definitions in the general case can be found in [3]. In this article we only need the summability of evaluations $\langle f(\mathbf{x}), \phi(\mathbf{x})\rangle$ where $f$ is a Radon measure. In this situation the evaluation $F(A)=\langle f(\mathbf{x}), \phi(\mathbf{x}) H(A-|\mathbf{x}|)\rangle$ is defined for any $A>0$, where $H(x)$ is the Heaviside function $(H(x)=0, x<0 ; H(x)=1, x>1)$, so that $H(A-|\mathbf{x}|)$ is the characteristic function of a ball of radius $A$. The evaluation $\langle f(\mathbf{x}), \phi(\mathbf{x})\rangle$ is Cesàro summable by spherical means if the limit of $F(A)$ as $A \rightarrow \infty$ exists in the Cesàro sense. We have:

Theorem 2.2. Let $f \in \mathcal{K}^{\prime}\left(\mathbb{R}^{d}\right)$ and $\phi \in \mathcal{K}(\mathbb{R})$. Then the evaluation $\langle f(\mathbf{x}), \phi(\mathbf{x})\rangle$ is Cesàro summable by spherical means.

Proof. See [3].

\section{The test in one VARIABle}

In this section we show that the integral test for the Cesàro summability of the series $\sum_{k=1}^{\infty} \phi(k)$ holds if $\phi \in \mathcal{G}_{\beta}(\mathbb{R})$ for some $\beta$ with $0<\beta \leq 1$.

Lemma 3.1. Suppose $\beta \leq 1$. Let $f \in \mathcal{G}_{\beta}^{\prime}(\mathbb{R})$ and $\phi \in \mathcal{G}_{\beta}(\mathbb{R})$. Then the evaluation $\langle f(x), \phi(x)\rangle$ is $(C)$ summable.

Proof. Since $\langle f(x), \phi(x)\rangle=\langle\phi(x) f(x), 1\rangle$, then in view of Theorem 2.1, it suffices to see that $\phi f \in \mathcal{K}^{\prime}$ whenever $f \in \mathcal{G}_{\beta}^{\prime}$ and $\phi \in \mathcal{G}_{\beta}$. But since $\langle\phi f, \psi\rangle=\langle f, \psi \phi\rangle$, this would follow if we show that $\psi \phi \in \mathcal{G}_{\beta}$ whenever $\psi \in \mathcal{K}$ and $\phi \in \mathcal{G}_{\beta}$. Now, if $\psi \in \mathcal{K}$, then $\psi^{(n)}(x)=O\left(|x|^{q-n}\right)$ as $|x| \rightarrow \infty$ for some $q \in \mathbb{R}$, while if $\phi \in \mathcal{G}_{\beta}$, then $\phi^{(n)}(x)=O\left(|x|^{p-n \beta}\right)$ as $|x| \rightarrow \infty$ for some $p \in \mathbb{R}$. Hence

$$
\begin{aligned}
(\psi \phi)^{(n)}(x) & =\sum_{k=0}^{n}\left(\begin{array}{l}
n \\
k
\end{array}\right) \psi^{(k)}(x) \phi^{(n-k)}(x) \\
& =\sum_{k=0}^{n} O\left(|x|^{q-k}\right) O\left(|x|^{p-\beta(n-k)}\right) \\
& =O\left(|x|^{q+p-\beta n}\right),
\end{aligned}
$$

since $\beta \leq 1$. Thus $\psi \phi \in \mathcal{G}_{\beta}$.

Lemma 3.2. Suppose $\beta>0$. Let $f$ be a periodic distribution with zero mean. Then $f \in \mathcal{G}_{\beta}^{\prime}$. 
Proof. Let $f_{n}$ be the $n$-th order primitive of $f$ with zero mean. If $n$ is large enough, then $f_{n}$ is continuous and, consequently, bounded on the whole real line. Let $\phi \in \mathcal{G}_{\beta}$. There exists $p \in \mathbb{R}$ such that $\phi^{(n)}(x)=O\left(|x|^{p-n \beta}\right)$ as $|x| \rightarrow \infty$. If $n$ is large enough, then $\langle f(x), \phi(x)\rangle=(-1)^{n}\left\langle f_{n}(x), \phi^{(n)}(x)\right\rangle$ is given by the convergent integral $(-1)^{n} \int_{-\infty}^{\infty} f_{n}(x) \phi^{(n)}(x) d x$.

If we apply the lemma to the distribution

$$
\sigma(x)=\sum_{k=-\infty}^{\infty} \delta(x-k)-1,
$$

it follows that $\sigma \in \mathcal{G}_{\beta}^{\prime}$ if $\beta>0$. If we multiply by a smooth cutoff function $\rho$ that satisfies $\rho(x)=0$ for $x<\frac{1}{4}$ and $\rho(x)=1$ for $x>\frac{3}{4}$, it follows that $\sum_{k=1}^{\infty} \delta(x-k)-\rho(x)$ belongs to $\mathcal{G}_{\beta}^{\prime}$, and so the same is true of

$$
\sigma_{+}(x)=\sum_{k=1}^{\infty} \delta(x-k)-H(x-1)
$$

which differs from this by the addition of a compactly supported distribution.

With these preliminaries we can give our extension of the integral test.

Theorem 3.1. Suppose $0<\beta \leq 1$. Let $\phi \in \mathcal{G}_{\beta}(\mathbb{R})$. Then the series

$$
\sum_{k=1}^{\infty} \phi(k)
$$

and the integral

$$
\int_{1}^{\infty} \phi(x) d x
$$

are both $(C)$ summable or neither is $(C)$ summable.

Proof. The distribution $\sigma_{+}(x)=\sum_{k=1}^{\infty} \delta(x-k)-H(x-1)$ belongs to $\mathcal{G}_{\beta}^{\prime}$ because $\beta>0$ and, in view of Lemma 3.1, $\left\langle\sigma_{+}(x), \phi(x)\right\rangle$ is $(C)$ summable because $\beta \leq 1$. That is, the Cesàro limit

$$
\lim _{A \rightarrow \infty}\left\langle\sigma_{+}(x) H(A-x), \phi(x)\right\rangle=\lim _{A \rightarrow \infty}\left(\sum_{k=1}^{\llbracket A \rrbracket} \phi(k)-\int_{1}^{A} \phi(x) d x\right)(C)
$$

exists. But since $\sum_{1 \leq k \leq A} \phi(k)=\left\langle\sigma_{+}(x) H(A-x), \phi(x)\right\rangle+\int_{1}^{A} \phi(x) d x$, the existence of the $(C)$ limit of $\sum_{1 \leq k \leq A} \phi(k)$ as $A \rightarrow \infty$ is equivalent to the existence of the (C) limit of $\int_{1}^{A} \phi(x) d x$ as $A \rightarrow \infty$.

Let us consider some illustrations.

Example 3.1. Let $\alpha$ be a constant that satisfies $0<\alpha<1$. Consider a smooth function that satisfies $\phi(x)=\frac{\sin \left(a x^{\alpha}\right)}{x}$ for $|x| \geq 1$. Then $\phi$ belongs to $\mathcal{G}_{1-\alpha}$. The integral $\int_{1}^{\infty} \phi(x) d x$ converges. It follows from the integral test of Theorem 3.1 that the series $\sum_{n=1}^{\infty} \frac{1}{n} \sin \left(a n^{\alpha}\right)$ is $(C)$ summable for any $a \in \mathbb{R}$. It then follows from a theorem of Littlewood [11] that the series is convergent: indeed, any Cesàro summable series is Abel summable and Littlewood's theorem says that if $\sum_{n=1}^{\infty} a_{n}$ is Abel summable and $a_{n}=O(1 / n)$, then $\sum_{n=1}^{\infty} a_{n}$ converges. 
The series $\sum_{n=1}^{\infty} \frac{1}{n} \sin \left(a n^{\alpha}\right)$ is not convergent if $a \in \mathbb{C} \backslash \mathbb{R}$, but the function $\Lambda$ defined by $\Lambda(a)=\sum_{n=1}^{\infty} \frac{1}{n} \sin \left(a n^{\alpha}\right), a>0$, admits a continuation to $\mathbb{C}$ as an entire function. It can be shown [1], [3] that

$$
\sum_{n=1}^{\infty} \frac{\sin \left(a n^{\alpha}\right)}{n}=\frac{\pi}{2 \alpha}+\sum_{k=0}^{\infty} \frac{(-1)^{k} a^{2 k+1} \zeta(1-(2 k+1) \alpha)}{(2 k+1) !}
$$

where $\zeta(s)$ is Riemann's zeta function.

Example 3.2. Observe that the integral $\int_{1}^{\infty} x^{\rho} \sin x d x$ is $(C)$ summable for any $\rho \in \mathbb{C}$. This can be seen by repeated integration by parts or by using Theorem 2.1 with $f(x)=H(x-1) x^{\rho} \sin x$, which belongs to $\mathcal{K}^{\prime}$. Thus, the integral $\int_{1}^{\infty} x^{\beta} \sin \left(a x^{\alpha}\right) d x$, which can be reduced to that one by a change of variables, is $(C)$ summable for any $\alpha>0$ and $\beta \in \mathbb{C}$. If $0<\alpha<1$, we can apply our test to a smooth function $\phi$ that satisfies $\phi(x)=x^{\beta} \sin \left(a x^{\alpha}\right)$ for $|x| \geq 1$, and which therefore belongs to $\mathcal{G}_{1-\alpha}$, to obtain that the series

$$
\sum_{n=1}^{\infty} n^{\beta} \sin \left(a n^{\alpha}\right)
$$

is $(C)$ summable for any $a \in \mathbb{R}, \beta \in \mathbb{C}$ if $0<\alpha<1$. Another proof can be seen in Hardy's book [8].

The test can also be applied to series containing Bessel functions.

Example 3.3. Let $J_{\nu}(x)$ be the Bessel function of the first kind of order $\nu \in \mathbb{R}$. If $0<\alpha<1$ and $\beta \in \mathbb{C}$, then a smooth function $\phi$ that satisfies $\phi(x)=|x|^{\beta} J_{\nu}\left(a|x|^{\alpha}\right)$ for $|x| \geq 1$ belongs to $\mathcal{G}_{1-\alpha}$. The integral $\int_{1}^{\infty} x^{\rho} J_{\nu}(x) d x$ is $(C)$ summable for any $\rho \in \mathbb{C}$, and so the same is true of the integral $\int_{1}^{\infty} x^{\beta} J_{\nu}\left(a x^{\alpha}\right) d x$ if $\beta \in \mathbb{C}, a \in \mathbb{R}$ and $\alpha>0$.

Hence, the series

$$
\sum_{n=1}^{\infty} n^{\beta} J_{\nu}\left(a n^{\alpha}\right)
$$

is Cesàro summable if $\beta \in \mathbb{C}, a \in \mathbb{R}$ and $0<\alpha<1$.

The standard integral test is also useful when both the series and the integral diverge. Indeed, if $\phi$ is positive and decreasing, then the sequence $\sum_{k=1}^{n} \phi(k)-$ $\int_{1}^{n} \phi(x) d x$ is also positive and decreasing and thus convergent. Thus $\sum_{k=1}^{n} \phi(k) \sim$ $\int_{1}^{n} \phi(x) d x+C$ for some constant $C$ as $n \rightarrow \infty$. In our case a similar result holds.

Example 3.4. Consider the function $\phi(x)=1$, which belongs to $\mathcal{G}_{1}=\mathcal{K}$. In this case both $\sum_{1 \leq k \leq A} 1=\llbracket A \rrbracket$ and $\int_{1}^{A} d x=A-1$ diverge to infinity as $A \rightarrow \infty$. Observe that

$$
\left\langle\sigma_{+}(x), \phi(x)\right\rangle=\frac{1}{2}
$$

since $\lim _{A \rightarrow \infty}(\llbracket A \rrbracket-(A-1))=\frac{1}{2}(C)$. Thus

$$
\sum_{1 \leq k \leq A} \phi(k) \sim \int_{1}^{A} \phi(x) d x+\frac{1}{2}(C) \quad \text { as } A \rightarrow \infty .
$$

Notice that if we set $A=N \in \mathbb{N}$, then $\lim _{N \rightarrow \infty}\left(\sum_{k=1}^{N} \phi(k)-\int_{1}^{N} \phi(x) d x\right)=1$, not $\frac{1}{2}$ as in (3.7). In general, if the real variable limit $L=\lim _{A \rightarrow \infty} f(A)(C)$ exists, 
then the Cesàro limit of a sequence $\left\{f\left(k_{n}\right)\right\}_{n=0}^{\infty}$, where $k_{n} \nearrow \infty$, may or may not exist; and even if it does, it might not equal $L$.

\section{THE TEST IN SEVERAL VARIABLES}

The results corresponding to Lemmas 3.1 and 3.2 remain valid in $\mathbb{R}^{d}$.

Lemma 4.1. Suppose $\beta \leq 1$. Let $f \in \mathcal{G}_{\beta}^{\prime}\left(\mathbb{R}^{d}\right)$ and $\phi \in \mathcal{G}_{\beta}\left(\mathbb{R}^{d}\right)$. Then the evaluation $\langle f(\mathbf{x}), \phi(\mathbf{x})\rangle$ is $(C)$ summable by spherical means.

Proof. As in the one-dimensional case, it suffices to verify that if $\beta \leq 1$, then $\phi \psi \in \mathcal{G}_{\beta}\left(\mathbb{R}^{d}\right)$ whenever $\phi \in \mathcal{G}_{\beta}\left(\mathbb{R}^{d}\right)$ and $\psi \in \mathcal{K}\left(\mathbb{R}^{d}\right)$.

In order to give our next result, we need to clarify the notion of mean of a periodic function of several variables. If $f \in \mathcal{D}^{\prime}\left(\mathbb{R}^{d}\right)$ satisfies $f\left(\mathbf{x}+\mathbf{e}_{j}\right)=f(\mathbf{x})$, $1 \leq j \leq d$, where $\mathbf{e}_{j}$ are the unit coordinate vectors, $\mathbf{e}_{j}=\left(\delta_{i j}\right)_{i=1}^{n}$, then $f$ admits an expansion in a Fourier series, $f(\mathbf{x})=\sum_{\mathbf{k} \in \mathbb{Z}^{d}} a_{\mathbf{k}} e^{2 \pi i \mathbf{x} \cdot \mathbf{k}}$. The mean of $f$ is the constant term in the Fourier series, namely, $a_{\mathbf{0}}$.

In general, $f \in \mathcal{D}^{\prime}\left(\mathbb{R}^{d}\right)$ is said to be periodic if the set of periods $P=\left\{\mathbf{p} \in \mathbb{R}^{d}\right.$ : $f(\mathbf{x}+\mathbf{p})=f(\mathbf{x})$ for all $\left.\mathbf{x} \in \mathbb{R}^{d}\right\}$ generates $\mathbb{R}^{d}$. If that is the case, then we can find a nonsingular linear transformation $A: \mathbb{R}^{d} \rightarrow \mathbb{R}^{d}$ such that $f(A \mathbf{x})$ has periods $\mathbf{e}_{1}, \ldots, \mathbf{e}_{d}$. The mean of $f$ is then the mean of $f(A \mathbf{x})$.

Lemma 4.2. Suppose $\beta>0$. Let $f$ be a periodic distribution in $\mathbb{R}^{d}$ with zero mean. Then $f \in \mathcal{G}_{\beta}^{\prime}\left(\mathbb{R}^{d}\right)$.

Proof. Let $L$ be the differential operator $\partial^{d} / \partial x_{1} \ldots \partial x_{d}$. Let $f_{n}$ be the periodic generalized function with zero mean that satisfies $L^{n} f_{n}=f$. If $n$ is large enough, then $f_{n}$ is continuous, and thus bounded. If $\phi \in \mathcal{G}_{\beta}\left(\mathbb{R}^{d}\right)$, then there exists $q \in \mathbb{R}$ such that $\mathbf{D}^{\mathbf{k}} \phi(\mathbf{x})=O\left(|\mathbf{x}|^{q-\beta|\mathbf{k}|}\right)$ as $|\mathbf{x}| \rightarrow \infty$. Hence, if $n$ is large enough, $\langle f(\mathbf{x}), \phi(\mathbf{x})\rangle=\left\langle f_{n}(\mathbf{x}),(-1)^{n d} L^{n} \phi(\mathbf{x})\right\rangle$ is a convergent integral, namely, $(-1)^{n d} \int_{\mathbb{R}^{d}} f_{n}(\mathbf{x}) L^{n} \phi(\mathbf{x}) d \mathbf{x}$.

We apply this lemma to the distribution

$$
\sigma(\mathbf{x})=\sigma_{d}(\mathbf{x})=\sum_{\mathbf{k} \in \mathbb{Z}^{d}} \delta(\mathbf{x}-\mathbf{k})-1 .
$$

This distribution is periodic, with $\mathbb{Z}^{d}$ as its lattice of periods, and has zero mean. Thus $\sigma \in \mathcal{G}_{\beta}\left(\mathbb{R}^{d}\right)$ if $\beta>0$.

Theorem 4.1. Suppose $0<\beta \leq 1$. Let $\phi \in G_{\beta}\left(\mathbb{R}^{d}\right)$. Then the series

$$
\sum_{\mathbf{k} \in \mathbb{Z}^{d}} \phi(\mathbf{k})
$$

and the integral

$$
\int_{\mathbb{R}^{d}} \phi(\mathbf{x}) d \mathbf{x}
$$

are both Cesàro summable by spherical means or neither of them is.

Proof. In view of Lemmas 4.1, 4.2 and Theorem 2.2, the evaluation $\langle\sigma(\mathbf{x}), \phi(\mathbf{x})\rangle$ is Cesàro summable by spherical means when $0<\beta \leq 1$. But since

$$
\langle\sigma(\mathbf{x}), \phi(\mathbf{x})\rangle=\lim _{A \rightarrow \infty}\left(\sum_{|\mathbf{k}| \leq A} \phi(\mathbf{k})-\int_{|\mathbf{x}| \leq A} \phi(\mathbf{x}) d \mathbf{x}\right)(C),
$$


it follows that (4.2) and (4.3) are both summable by spherical means or neither is.

Let us consider some illustrations.

Example. Let $\alpha_{1}, \ldots, \alpha_{d}$ be numbers that satisfy $0<\alpha_{j}<1,1 \leq j \leq d$. Let $\phi$ be a smooth function that satisfies

$$
\begin{aligned}
& \phi\left(x_{1}, \ldots, x_{d}\right) \\
& \quad=p\left(x_{1}, \ldots, x_{d}\right) \sin \left(a_{1}\left|x_{1}\right|^{\alpha_{1}}+a_{2}\left|x_{2}\right|^{\alpha_{2}}+\cdots+a_{d}\left|x_{d}\right|^{\alpha_{d}}\right) \quad \text { for }\left|x_{j}\right| \geq 1,
\end{aligned}
$$

where $p$ is a polynomial. Then $\phi$ belongs to $\mathcal{G}_{1-\alpha}$, where $\alpha=\max \left\{\alpha_{1}, \ldots, \alpha_{d}\right\}$. It is easy to see that the integral $\int_{\mathbb{R}^{d}} \phi(\mathbf{x}) d \mathbf{x}$ is $(C)$ summable by spherical means. Thus, the series

$$
\sum_{\mathbf{k} \in \mathbb{Z}^{d}} p\left(k_{1}, \ldots, k_{d}\right) \sin \left(a_{1}\left|k_{1}\right|^{\alpha_{1}}+a_{2}\left|k_{2}\right|^{\alpha_{2}}+\cdots+a_{d}\left|k_{d}\right|^{\alpha_{d}}\right)
$$

is Cesàro summable by spherical means.

Example. If $0<\alpha<1, \nu \in \mathbb{R}$ and $\beta \in \mathbb{R}$, then in $\mathbb{R}^{d}$ the integral

$$
\int_{|\mathbf{x}| \geq 1}|\mathbf{x}|^{\beta} J_{\nu}\left(|\mathbf{x}|^{\alpha}\right) d \mathbf{x}
$$

is Cesàro summable. Therefore, the series

$$
\sum_{\mathbf{k} \in \mathbb{Z}^{d} \backslash\{\mathbf{0}\}}|\mathbf{k}|^{\beta} J_{\nu}\left(|\mathbf{k}|^{\alpha}\right)
$$

is Cesàro summable.

\section{REFERENCES}

1. J. Boersma, A nonharmonic trigonometric series, solution of Problem 94-12, SIAM Review 37 (1995), 443-445.

2. A. L. Durán and R. Estrada, The analytic continuation of moment functions and of zeta functions, preprint, San José, 1997.

3. A. L. Durán, R. Estrada and R. P. Kanwal, Extensions of the Poisson summation formula, J. Math. Anal. Appl. 218 (1998), 581-606. CMP 98:08

4. R. Estrada, The Cesàro behavior of distributions, Proc. Roy. Soc. London A (to appear).

5. R. Estrada, J. M. Gracia-Bondía and J. C. Várilly, On summability of distributions and spectral geometry, Commun. Math. Phys. 191 (1998), 219-248. CMP 98:07

6. R. Estrada and R. P. Kanwal, Asymptotic Analysis: a Distributional Approach, Birkhäuser, Boston, 1994. MR 95g:46071

7. A. Grossmann, G. Loupias and E. M. Stein, An algebra of pseudodifferential operators and quantum mechanics in phase space, Ann. Inst. Fourier 18 (1968), 343-368. MR 42:2327

8. G. H. Hardy, Divergent Series, Clarendon Press, Oxford, 1949. MR 11:25a

9. R. P. Kanwal, Generalized Functions: Theory and Technique, Academic Press, New York, 1983. MR 85f:46001

10. L. Schwartz, Théorie des Distributions, Hermann, Paris, 1966. MR 35:730

11. E. C. Titchmarsh, The Theory of Functions, Oxford University Press, Oxford, 1939.

Escuela de Matemática, Universidad de Costa Rica, 2060 San José, Costa Rica

E-mail address: analiad@emate.ucr.ac.cr

E-mail address: restrada@cariari.ucr.ac.cr 